\title{
Case Report: Isolated Pauci-immune Vasculitis of the Pituitary Gland Revealed by Stereotactic Biopsy
}

\author{
One Sentence Summary: The diagnosis of a pauci-immune vasculitis of the \\ pituitary without systemic disease was established by stereotactic biopsy in a \\ 36-year-old man.
}

Authors

Affiliations
R. Roelz ${ }^{1,2}$, D. Erny ${ }^{3}$, M. J. Shah ${ }^{1}$, K. Egger ${ }^{4}$, J. Thiel ${ }^{5}$, J. Seufert ${ }^{6}$, K. Laubner ${ }^{6}$, P. Reinacher ${ }^{2}$

Affiliation addresses are listed at the end of the article

\author{
Key words \\ vasculitis \\ - pituitary \\ - GPA
}

received $\quad 27.10 .2014$

first decision 09.03.2015

accepted 16.03.2015

Bibliography

DOI http://dx.doi.org/

10.1055/s-0035-1548827

Exp Clin Endocrinol Diabetes

Rep 2015; 2: e4-e7

(C) J. A. Barth Verlag in

Georg Thieme Verlag KG

Stuttgart · New York

ISSN 2196-7407

\section{Correspondence \\ Dr. R. Roelz}

Department of Neurosurgery

University Medical Center

Freiburg

Breisacher Str. 64

79106 Freiburg

Germany

Tel.: $+49 / 761 / 27050010$

Fax: + 49/761/270 93090

roland.roelz@uniklinik-freiburg.

de

\section{License terms}

\section{(๑) $\Theta \circledast$}

\section{Abstract}

V

Purpose: To demonstrate a case of isolated pauci-immune vasculitis of the pituitary gland in which the diagnosis was attained by stereotactic biopsy.

Methods: We report a 36-year-old man who presented with diabetes insipidus and secondary hypogonadism. Cranial MRI revealed an enhancing lesion of the pituitary stalk. A histological sample was obtained by stereotactic biopsy and pathological work-up provided the diagnosis of isolated pauci-immune vasculitis. No further organ manifestations or serologic signs of systemic vasculitis were identified. There was no response to immunosuppressive therapy and the patient refused treatment with Rituximab.

Conclusions: Vasculitis of the pituitary gland is a rare condition. Less than 50 cases have been described to date in patients with granulomatosis with polyangiitis (GPA, or Wegener's granulo-

\section{Case Report}

$\nabla$

A 36-year-old man was admitted to our hospital with polyuria and polydipsia, mild headaches, dizziness, fatigue and general weakness since 4 weeks. There were no other specific symptoms reported by the patient. His medical history did not reveal any previous diseases or disorders. Physical and neurological examinations were adequate.

The polyuria and polydipsia in combination with low urine osmolality (102 mosm $/ \mathrm{kg}$ ) were highly suggestive of diabetes insipidus (DI). Therefore, a water restriction test was performed. It was terminated after $12 \mathrm{~h}$ due to urine output of $5 \mathrm{l}$ and $7 \%$ loss of body weight. With water deprivation, diuresis and low urine osmolality persisted (105 mosmol/kg at baseline and $214 \mathrm{mosmol} / \mathrm{kg}$ matosis) and few case reports exist on pituitary involvement in other systemic vasculitis like Behcet's disease and Cogan's syndrome [1-9]. Pituitary involvement in GPA predominantly affects the posterior pituitary gland resulting in central diabetes insipidus (DI) but global or partial anterior pituitary dysfunction and compression of the optic chiasm has also been described (10). Relapse and permanent residual pituitary insufficiency is common despite immunosuppressive therapy [7]. Vasculitis of the pituitary gland without manifestation in other organs has been described in few patients and diagnosis of GPA was attained by the presence of proteinase-3 specific ANCA and/or transsphenoidal biopsy in these cases [10-12].

The case presented here demonstrates that pauci-immune vasculitis of the pituitary can occur in the absence of systemic disease. The diagnosis can be made by stereotactic biopsy but therapy remains a challenge.

at termination) and serum sodium levels rose from $145 \mathrm{mmol} / \mathrm{l}$ to $154 \mathrm{mmol} / \mathrm{l}$. Intranasal application of desmopressin $10 \mu \mathrm{g}$ normalized serum sodium as well as hourly urine output, urine osmolality rose to $518 \mathrm{mosmol} / \mathrm{kg}$ confirming the diagnosis of central DI. Urine routine tests and urinary sediment analysis did not show abnormalities. A diabetes mellitus type 2 was ruled out.

Assessment of his anterior pituitary function showed baseline 09:00 cortisol of $286 \mathrm{nmol} / \mathrm{l}$ and adrenocorticotropin of $16.4 \mathrm{pg} / \mathrm{ml}$ (RR: $7.2-$ $63.3 \mathrm{pg} / \mathrm{ml}$ ). An ACTH-test with Stimulation with $250 \mu$ g tetracosactrin demonstrated an increase in serum cortisol to $921 \mathrm{nmol} / 1$ indicating a normal adrenocortical reserve. A normal level of thyroid stimulating hormone (TSH) of $0.96 \mu \mathrm{U} / \mathrm{ml}$ (RR: $0.27-4.20 \mu \mathrm{U} / \mathrm{ml}$ ) and free thyroxin (fT4) 
10.7 (RR: 10.6-22.7 pmol/l) was documented. Triiodothyronine (fT3) was low at $3.11 \mathrm{pmol} / \mathrm{l}$ (RR: 3.4-6.8 pmol/l). Regular thyreotropic function was confirmed by a TRH (thyrotropin releasing hormone) test: Intravenous administration of $200 \mu \mathrm{g}$ TRH increased the baseline TSH from $1.44 \mu \mathrm{U} / \mathrm{ml}$ to $7.09 \mu \mathrm{U} / \mathrm{ml}$ after $30 \mathrm{~min}$. The morning testosterone level was low in repeated measurements (6.98-8.13 nmol/l, RR: $8.64-29.0 \mathrm{nmol} / \mathrm{l}$ ) and partial insufficiency of the gonadotropic axis was proven by a GnRH-test (gonadotropin-releasing hormone). $100 \mu \mathrm{g}$ of intravenous GnRH prompted an adequate increase of luteinizing hormone (LH) from 3.5 to $11.4 \mathrm{mIU} / \mathrm{ml}$ (RR: $1.7-8.6 \mathrm{mIU} / \mathrm{ml}$ ) after $30 \mathrm{~min}$. The increment of testosterone $(6.17-6.01 \mathrm{nmol} /, \mathrm{RR}$ : 8.64-29.0 nmol/l) and follicle stimulating hormone (FSH) (1.6$2.2 \mathrm{mIU} / \mathrm{ml}$, RR: $1.5-12.4 \mathrm{mIU} / \mathrm{ml}$ ) after $30 \mathrm{~min}$ was low. Substitution of testosterone $25 \mathrm{mg}$ daily was initiated. Prolactin was within normal range with $286 \mu \mathrm{IU} / \mathrm{ml}$ (RR: $86-324 \mu \mathrm{IU} / \mathrm{ml}$ ), IGF-1 was also in normal range with $163 \mathrm{ng} / \mathrm{ml} / \mathrm{RR} 109-284 \mathrm{ng} / \mathrm{ml}$ ).

Serologic testing for antinuclear antibodies (ANA), anti-double stranded DNA (anti-dsDNA) antibodies, anti-phospholipid antibodies, myeloperoxidase- and proteinase 3 specific anti-neutrophil cytoplasmic antibodies (ANCA) was negative. Complement components C3, C4, and complement split product C3d were within normal ranges. Total hemolytic complement activity measured by $\mathrm{CH} 50$ was within normal limits. A high resolution computed tomography of the chest showed no abnormalities. Thus, a diagnosis of a systemic vasculitis, especially GPA (Wegener's granulomatosis) in which pituitary involvement has been described before, was unlikely. Analysis of cerebrospinal fluid (CSF) obtained by lumbar puncture showed a cell count of 2 per $\mu$ with $90 \%$ lymphocytes, $10 \%$ monocytes and an elevated CD4/ CD8 ratio of 5.3. Protein $(630 \mathrm{mg} / \mathrm{l})$ and lactate $(1.36 \mathrm{mmol} / \mathrm{l}) \mathrm{lev}-$ els were marginally higher than normal. There were no signs of local synthesis of immunoglobulins. The patients' serum showed normal Interleukin-2 receptor $(266 \mathrm{U} / \mathrm{ml})$, neopterin $(5.6 \mathrm{nmol} / \mathrm{l})$ and angiotensin-converting enzyme $(28.7 \mathrm{U} / \mathrm{l})$ concentrations making a diagnosis of neurosarcoidosis improbable.

A cranial MRI revealed a single Gadolinium-enhancing nodule of $8 \times 6 \times 7 \mathrm{~mm}$ at the pituitary stalk ( $\odot$ Fig. 1 ). No abnormalities of the paranasal sinuses were present. A stereotactic biopsy via a right frontal trajectory was performed using a Leksell stereotaxy system and a $2.1 \mathrm{~mm}$ side-cutting biopsy needle.

Hematoxylin-eosin stained formalin-fixed and paraffin-embedded sections of the stereotactic biopsy material showed dense perivascular as well as intraparenchymal mononuclear infiltrates ( $\bullet$ Fig. 2a, b). These infiltrates were embedded in a loose glial matrix exhibiting distinct astrogliosis as seen in the GFAP stain ( $\bullet$ Fig. 2c). The infiltrates themselves contained mainly

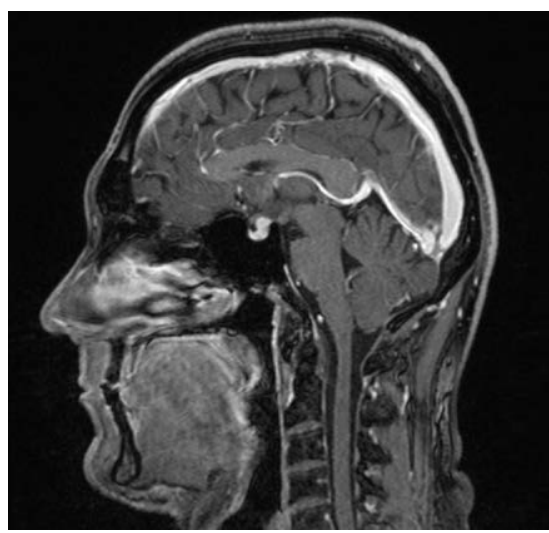

Fig. 1 Contrastenhanced T1w sagittal MRI image through the pituitary gland shows a Gadolinium-enhancing nodule at the dorsal aspect of the pituitary stalk. mature lymphocytes, mostly with scarce cytoplasm and round, hyperchromatic nuclei, exhibiting CD3 and CD4 immunoreactivity ( $\odot$ Fig. 2d, e) and several CD20-positive B cells ( $\odot$ Fig. 2f). Only single CD8-positive cells could be detected within the infil-

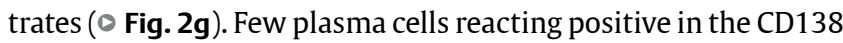
immunohistochemical staining as well as immature B cells staining positive for CD79a were observed. Several CD68-positive macrophages were present. Staining for components of the complement system (C1q, C3c, C4d, C5b-9), IgG and IgG4 subclass were negative (not shown). The small blood vessel walls were fragmented, a characteristic hallmark of vasculitis, as seen clearly in the Tibor Pap Silver staining ( $\bullet$ Fig. $\mathbf{2 h}$ ). Cloddy myelin loss ( $\odot$ Fig. $\mathbf{2 i}$ ) as well as axonal damage could be detected in the myelin basic protein (MBP) and Bielschowsky silver staining, respectively. Mitotic figures were absent and MIB1 staining (against the Ki-67 antigen), in which only few hematopoietic cells were marked positively, confirmed low proliferative activity. Special stains for the protozoan Toxoplasma gondii and various viruses (i.e., EBV, CMV, HSV1 and HSV2) were negative in the available specimens. Pituitary gland tissue was not detectable in the stereotactic biopsy material.

In summary, the histomorphological as well as immunohistochemical findings allowed for the diagnosis of vasculitis accompanied by secondary myelin loss and axonal damage. There was no evidence of a malignant neoplastic proliferative process.

A treatment with methylprednisolone and azathioprine (AZA) was initiated and AZA was gradually increased to a daily dose of $150 \mathrm{mg}$ per day. MRI follow-up 10 weeks after AZA had been started showed no change of the lesion at the pituitary stalk. As we were not sure whether AZA was given long enough to reach its effect methylprednisolone dose was increased to $1 \mathrm{mg} / \mathrm{kg}$ for one week and then tapered within 5 weeks to a maintenance dose of $5 \mathrm{mg} / \mathrm{d}$. However, MRI follow-up 6 and 8 months after diagnosis showed no response. We advocated a change of the therapeutic regimen to Rituximab but the patient refused further treatment other than hormone replacement. Diabetes insipidus and secondary hypogonadism persisted, but the patient did well under hormone replacement therapy with testosterone and desmopressin.

\section{Discussion}

\section{$\nabla$}

Pituitary involvement in systemic vasculitis is extremely rare and most cases have been described in GPA [3-5, 7, 10,13,14]. Predominantly the posterior pituitary is affected causing central DI but partial or total anterior pituitary dysfunction can be present. Although the pituitary can be the first organ affected by GPA and diabetes insipidus may be the presenting symptom, systemic involvement was present or ensued in all cases of pituitary vasculitis reported to date $[7,15]$. The patient presented here is, to the best of our knowledge, the first reported case of a vasculitis of the pituitary without systemic disease. Lack of immune-complex deposition ("pauci-immune") is typical for the ANCA-associated vasculitides like GPA or MPA. As in the patient we report a pauci-immune vasculitis was diagnosed on histologic examination, we suspect a localized form of GPA or MPA in the patient. The patient was ANCA negative, but localized disease stages of GPA and MPA often lack ANCAs in peripheral blood. The differential diagnoses for pituitary gland lesions are broad. Pituitary gland lesions can take both an expansive and/or invasive course. As destruction of the surrounding tissue 

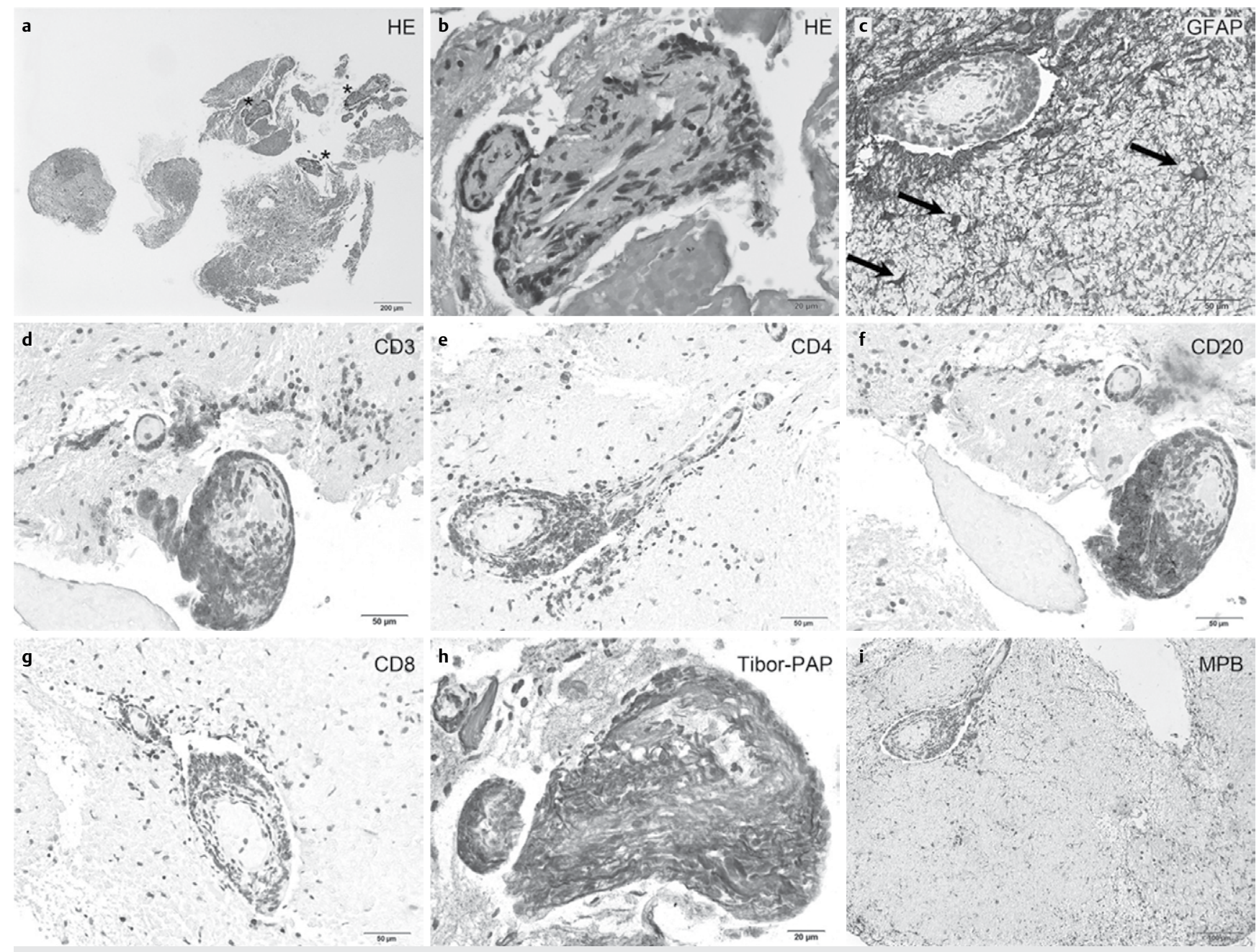

Fig. 2 Hematoxylin-eosin-stained sections (40 and 400 fold magnification) revealing dense perivascular mononuclear infiltrates within a loose glial matrix a, b which exhibits marked astrogliosis as seen in the GFAP (Glial fibrillary acidic protein) stain c. Immunohistochemical staining, at 200 fold magnification, clearly shows that the perivascular infiltrates consist mostly of T cells staining positive for CD3 and CD4 d, e as well as several CD20-positive B cells f. Only single CD8-positive cells are marked within the infiltrates $\mathbf{g}$. Tibor Pap silver staining at 400 fold magnification detecting fragmented endothelium of the vessels around which the mononuclear infiltrates are centered. Cloddy myelin loss can be detected in the myelin basic protein (MBP) staining at $100 \mathrm{fold}$ magnification i. Size bars $=200 \mu \mathrm{m}, 100 \mu \mathrm{m}, 50 \mu \mathrm{m}$ and $20 \mu \mathrm{m}$.

rapidly leads to severe neurological deficits (vision loss, anterior pituitary insufficiency) initiation of the correct treatment is of outmost importance. We demonstrate here that it is feasible to establish the diagnosis of an isolated vasculitis of the pituitary stalk by stereotactic biopsy. The patient was started on a combined immunosuppressive treatment with azathioprine and glucocorticoids. However, follow-up MRI 6 and 8 months after diagnosis showed no response. Kapoor et al. recently reported good remission rates of DI and poor remission rates for anterior pituitary function in their series of 8 patients. Interestingly, this was independent from morphological response of the pituitary lesions on MRI [10]. In line with most published cases, our patient did not regain pituitary function as DI and secondary hypogonadism persisted [7]. Unfortunately, the patient is refusing a therapy with the B-cell depleting monoclonal antibody Rituximab (RTX). RTX has been proven to be effective in ANCAassociated vasculitis in a randomized, placebo-controlled trial and was effective in the treatment of several patients with pituitary involvement of GPA that did not respond to cyclophosphamide $[10,13,14]$.

\section{Conclusion}

$\nabla$

Vasculitis of the pituitary gland causing pituitary insufficiency was previously reported as a rare manifestation of systemic vasculitis, mainly in GPA. Serologic markers and the presence of further organ involvement can help to establish the diagnosis in systemic vasculitis. Unfortunately, diagnosing a limited, localized variant of vasculitides remains extremely challenging. The case reported here demonstrates that isolated vasculitis of the pituitary can occur in the absence of systemic disease. In this setting, stereotactic biopsy can pave the way to diagnosis and help to timely initiate therapy. Azathioprine and steroids did not induce remission in our patient. We strongly suggest a therapy with Rituximab.

\section{Disclosure and Funding \\ $\nabla$}

The authors declare no conflict of interest and disclose no funding for the present article. 
Disclosure statement: The authors have nothing to disclose.

\section{Affiliations}

${ }^{1}$ Department of Neurosurgery, University Medical Center Freiburg,

Freiburg, Germany

${ }^{2}$ Department of Stereotactic and Functional Neurosurgery, University

Medical Center Freiburg, Freiburg, Germany

${ }^{3}$ Department of Neuropathology, University Medical Center Freiburg,

Freiburg, Germany

${ }^{4}$ Department of Neuroradiology, University Medical Center Freiburg, Freiburg, Germany

${ }^{5}$ Department of Rheumatology and Clinical Immunology, University Medical

Center Freiburg, Freiburg, Germany

${ }^{6}$ Division of Endocrinology and Diabetology, Department of Internal

Medicine II, University Medical Center Freiburg, Freiburg, Germany

\section{References}

1 Kara O, Demirel F, Acar BC, Cakar N. Wegener granulomatosis as an uncommon cause of panhypopituitarism in childhood. J Pediatr Endocrinol Metab JPEM 2013; 26: 959-962

2 Slabu H, Arnason T. Pituitary granulomatosis with polyangiitis. BM] Case Rep 2013

3 Hughes J, Barkhoudarian G, Ciarlini P, Laws ER, Mody E, Inzucchi SE. Refractory pituitary granulomatosis with polyangiitis (Wegener's) treated with rituximab. Endocr Pract Off J Am Coll Endocrinol Am Assoc Clin Endocrinol 2013; 19: e1-e7
4 Santoro SG, Guida AH, Furioso AE, Glikman P, Rogozinski AS. Panhypopituitarism due to Wegener's granulomatosis. Arq Bras Endocrinol Metabol 2011; 55: 481-485

5 Tenorio Jimenez C, Montalvo Valdivieso A, López Gallardo G, Mcgowan $B$. Pituitary involvement in Wegener's granulomatosis: unusual biochemical findings and severe malnutrition. BMJ Case Rep 2011

6 Xue J, Wang $H, W u H$, Jin $Q$. Wegener's granulomatosis complicated by central diabetes insipidus and peripheral neutrophy with normal pituitary in a patient. Rheumatol Int 2009; 29: 1213-1217

7 Yong TY, Li JYZ, Amato L, Mahadevan K, Phillips PJ, Coates PS. Pituitary involvement in Wegener's granulomatosis. Pituitary 2008; 11: 77-84

8 Kanatani M, Nakamura $R$, Kurokawa K, Taoda M, Nemoto Y, Kamakura $K$. Hypopituitarism associated with Cogan's syndrome; high-dose glucocorticoid therapy reverses pituitary swelling. Jpn J Med 1991; 30: 164-169

9 Otsuka F, Amano T, Ogura T, Ota Z. Diabetes insipidus with Behçet's disease. Lancet 1995; 346: 1494-1495

10 Kapoor E, Cartin-Ceba R, Specks U, Leavitt J, Erickson B, Erickson D. Pituitary Dysfunction in Granulomatosis with Polyangiitis: The Mayo Clinic Experience. J Clin Endocrinol Metab 2014; jc20141962

11 Al-Fakhouri A, Manadan A, Gan J, Sreih AG. Central diabetes insipidus as the presenting symptom of granulomatosis with polyangiitis. J Clin Rheumatol Pract Rep Rheum Musculoskelet Dis 2014; 20: 151-154

12 Dutta P, Hayatbhat M, Bhansali A, Bambery P, Kakar N. Wegener's granulomatosis presenting as diabetes insipidus. Exp Clin Endocrinol Diabetes Off J Ger Soc Endocrinol Ger Diabetes Assoc 2006; 114: 533-536 